\title{
The Development of Beam Current Monitors in the APS*
}

\author{
$X$. Wang, F. Lenkszus, and E. Rotela \\ Advanced Photon Source, Argonne National Laboratory \\ 9700 South Cass Avenue, Argonne, Illinois 60439
}

\section{Abstract}

The Advanced Photon Source (APS) is a third-generation 7-GeV synchrotron radiation source. The precision measurement of beam current is a challenging task in high energy accelerators, such as the APS, with a wide range of beam parameters and complicated noise, radiation, and thermal environments. The beam pulses in the APS injector and storage ring have charge ranging from $50 \mathrm{pC}$ to $25 \mathrm{nC}$ with pulse durations varying from $30 \mathrm{ps}$ to $30 \mathrm{~ns}$. A total of nine non-intercepting beam current monitors have been installed in the APS facility (excluding those in the linac) for general current measurement. In addition, several independent current monitors with specially designed redundant interlock electronics are installed for personnel safety and machine protection. This paper documents the design and development of current monitors in the APS, discusses the commissioning experience in the past year, and presents the results of recent operations.

\section{INTRODUCTION}

The Advanced Photon Source, now in the commissioning phase, is a 7-GeV storage ring served by a full-energy injector consisting of an electron/positron linac, a positron accumulator ring (PAR), a $7-\mathrm{GeV}$ booster, and associated beam transfer lines.

Non-intercepting, high-accuracy beam current monitors are required for the measurement of total charge, peak current, average-current, lifetime, transfer efficiency, and absolute beam loss. Figure 1 shows the layout of the current monitors in the APS. All current monitors shown have been installed and used extensively to support APS commissioning and operation.

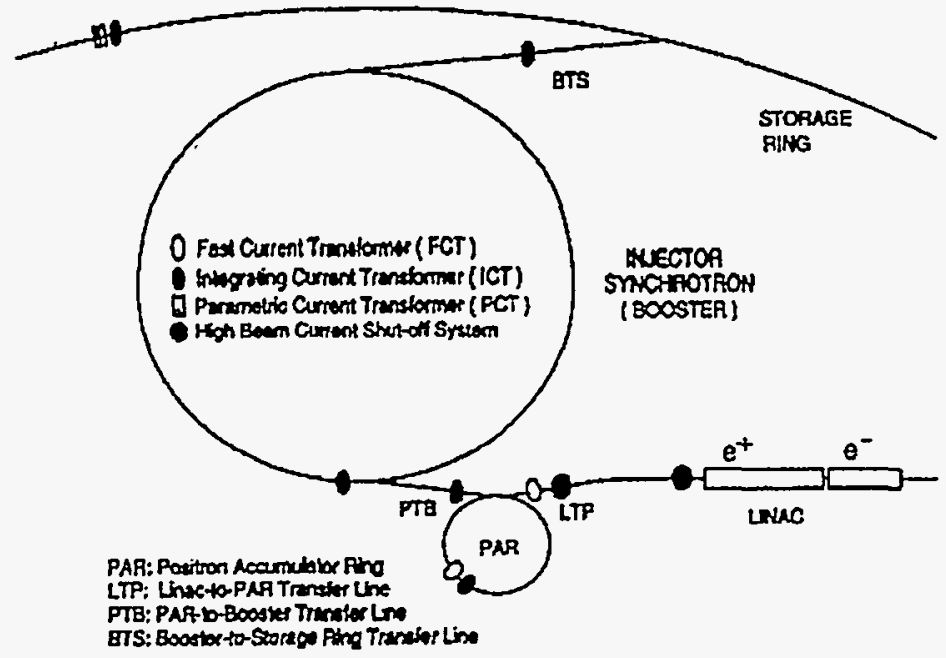

Figure 1: Layout of beam current monitors in the APS

* Work supported by U.S. Department of Energy. Office of Basic Sciences, under Contract No. W-31-109-ENG-38.

\section{INJECTOR CURRENT MONITORS}

The current monitors in the APS injector are primarily used for measuring total beam charge and transfer efficiency at various locations. The accelerator machine studies and operation require a non-intercepting device that (1) performs realtime measurements with high absolute accuracy, (2) is bipolar so that it can accommodate both electron and positron operations, and (3) has a wide dynamic range up to $\pm 30 \mathrm{nC}$ with a resolution better than $25 \mathrm{pC}$.

Monitoring of the beam charge/current is based on the use of integrating or fast current transformers (ICTs or FCTs) manufactured by Bergoz and in-house beam current monitoring electronics [1]. The FCT is a passive AC transformer with ins rise time. It is used to measure peak current and total charge of the linac 30ns macropulse in the linac-to-PAR (LTP) transfer line. The ICT is a capacitively shorted transformer [2] that stretches a beam bunch of a few tens of picoseconds to an output pulse of around 25 nanoseconds with its area proportional to the total charge of the beam bunch. The signal processing of the ICT output waveform requires a high precision measurement of the voltage $x$ time area of a very short pulse, which cannot be done by commercially available gated integrators using conventional designs. An ultrafast, high-precision gated integrator [3] has been developed to accurately calculate the transformer's output waveform area and to hold its output DC level proportional to the total beam charge for digitizing. This gated integrator provides fast response and high precision by introducing new design approaches. The various circuit errors usually associated with a high-speed gated integrator are virtually eliminated. Consequently, the relationship between the input and output of the integrator is clearly defined. The integrator is totally bipolar, resettable, and is capable of subtracting the input signal baseline offset within just one timing window, which has been proven extremely useful in processing the current transformer signals and achieving high noise rejection.

The current transformer inside the tunnel is connected to the nearest instrument room via a low-loss Heliax cable. The signal is fed into a single 6U VME current monitoring electronics module, which can be accessed by the control system. The main features of the VME module include a fast gated integrator with automatic baseline subtraction, completely programmable timing circuitry, and an on-board 12-bit A/D converter. The current monitor data acquisition is performed by the APS control system, which is a distributed system consisting of workstations, network servers, and IOCs. Associated operating programs are from the Experimental Physics and Industrial Control System (EPICS). The resulting digital data is converted to beam charge and current information and displayed on the workstation screen. The programs provide mouse-controlled operation for system setup and control. 


\section{DISCLAIMER}

Portions of this document may be illegible in electronic image products. Images are produced from the best available original document. 
The same system is used to measure the average current in the PAR and booster. Since there is only one beam bunch in the PAR or booster at a given time, the average current in the rings can be simply obtained by dividing the measured total charge of the single bunch by the ring revolution time.

To use the current monitor as a real-time tuning aid, the buffered ICT raw signals in the rings are made available in the main control room (MCR). For those ICTs too far away from the MCR, the signal is preserved by displaying it on an oscilloscope located in the nearby instrument room. The scope screen is observed by a charge-coupled device (CCD) camera and the video signal is transmitted by optical cable to a screen in the MCR. The scope can be accessed by the MCR via a GPIB bus and the setup can be adjusted on an EPICS screen.

The charge/current monitors described have all been installed and commissioned. Each system was calibrated prior to operation by injecting the test pulses to a single-turn calibration winding of the transformer to simulate the beam pulses of fixed charges. Timing variables for the gated integrator must also be set properly. The gate delay and width were adjusted for each monitor so that the beam pulse falls well within the gate window.

The PAR current monitor data is shown in Fig. 2. The ICT output signal shows a large signal baseline offset relative to the signal amplitude due to small pulse separation (102ns) in the PAR. The offset level changes as beam intensity changes. The high performance of the fast gated integrator is well demonstrated here in the signal bunch charge measurement. As shown in Fig. 2, the signal baseline is subtracted and only one beam bunch signal is integrated within the $100 \mathrm{~ns}$ gate window. The negative DC level (bottom trace) proportional to the total charge of single electron bunch is digitized for readout.

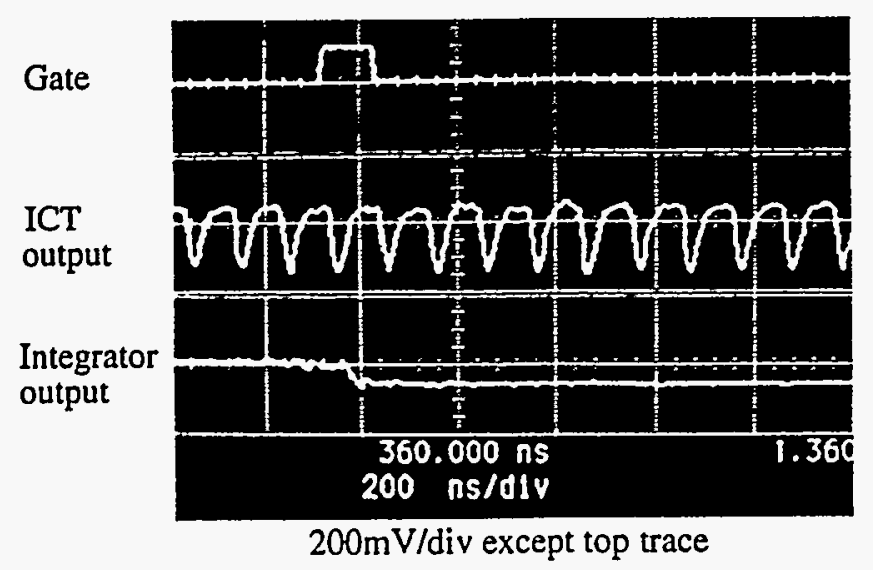

Figure 2: PAR current monitor signals

Figure 3 is an EPICS screen displaying the total beam charges at three different locations. In this case, two linac macrobunches were accumulated in the PAR and then extracted to the PTB transfer line for injection into the booster. The transfer efficiency is near $100 \%$.

Kicker noise has been identified as a major noise contributor to the current monitor signal due to the same arrival time and simple kicker shielding and grounding design. The current monitor system grounding and shielding method discussed in [4] has helped significantly to reduce the noise pickup. However, the noise level and appearance vary from kicker to kicker depending on the grounding structure and the physical location relative to a specific current transformer. Figure 4 shows the signal from the current monitor in the booster-to-storage ring (BTS) transfer line. The spike is the bunch signal. The underlying wave is noise pickup from the booster extraction kicker. The noise added about $300 \mathrm{pC}$ offset to the readout even though the signal was integrated within a very narrow gate window. The problem was resolved by increasing the baseline sampling speed in the gated integrator so that the underlying kicker noise could be subtracted as baseline offset.

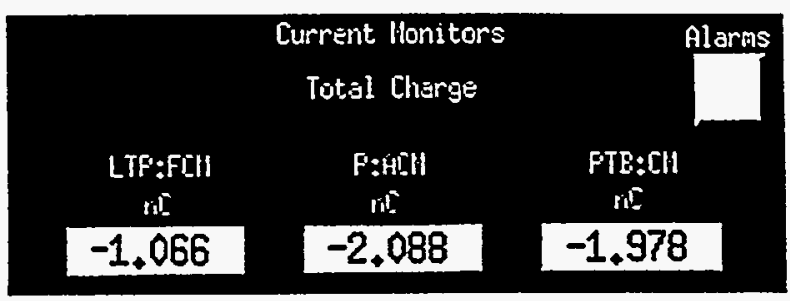

Figure 3: An EPICS screen showing total charges

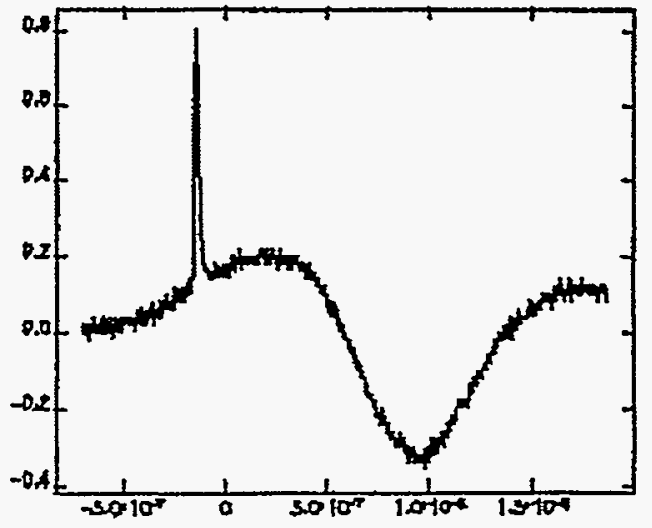

Figure 4: The BTS current monitor signal

The current monitors with specially designed interlock circuitry are also used to provide personnel safety protection in the event of acceleration of excessive beam currents. Two independent high beam current shut-off systems [5] have been installed in the linac and the LTP transfer line as shown in Fig. 1. Beam current is monitored by an FCT and fully redundant supervisory circuits connected to the Access Control Interlock System (ACIS) for beam intensity related shutdowns of the linac. To ensure a high degree of reliability, both systems employ a continuous self-checking function which injects a test pulse to a single-turn test winding after each beam pulse to verify that the system is fully functional. The system is designed to be fail-safe for all possible system faults such as loss of power, open or shorted signal or test cables, loss of external trigger, etc. The capability of the system allows management to define an operational safety envelope that contributes to significantly improved protection at the accelerator. 


\section{STORAGE RING CURRENT MONITORS}

Two current transformers are installed in the storage ring for both average current and injection charge measurements. Average current is measured by Bergoz's parametric current transformer (PCT) with resolution better than $5 \mu \mathrm{A}$ and $400 \mathrm{~mA}$ full scale range. The PCT output is digitized by a high-precision digital voltmeter and read out via GPIB. Single turn injection is monitored by an ICT and the VME module current monitoring electronics described above.

Two current transformers share the same housing and are located in the storage ring sector 35 straight section to avoid synchrotron radiation heating. Since the PCT is sensitive to the nearby stray magnetic fields which could add significant offset to the PCT output, the shield of the housing was very carefully designed. The transformers are completely surrounded by shields, composed of four layers of mu-metal and two layers of copper-plated low carbon steel for wide-range noise shielding. The vacuum chamber is formed by a commercial ceramic break and a welded bellows that protects the ceramic. To limit rf leakage and prevent the housing cavity from ringing with the beam frequency components, the ceramic break is wrapped with metal foil isolated by kapton tape overlapping the vacuum chamber on both sides of the break. In addition, a stainless steel tube with the same aperture as the storage ring vacuum chamber and a $2 \mathrm{~mm}$ gap at the center is suspended inside the ceramic break and bellows and attached to two end flanges. Total capacitance of the two gaps is around $450 \mathrm{pF}$. Other features of the housing assembly include two built-in heaters for bakeout, a water-cooled transformer support to prevent excessive heating from damaging the transformers, and a thermocouple probe for monitoring the air temperature around the transformers.

Figure 5 shows the PCT data of the APS first stored beam along with an exponential fit. The data fits the exponential extremely well. The monitor detected very low stored beam. No offset errors due to nearby stray magnetic field were observed.

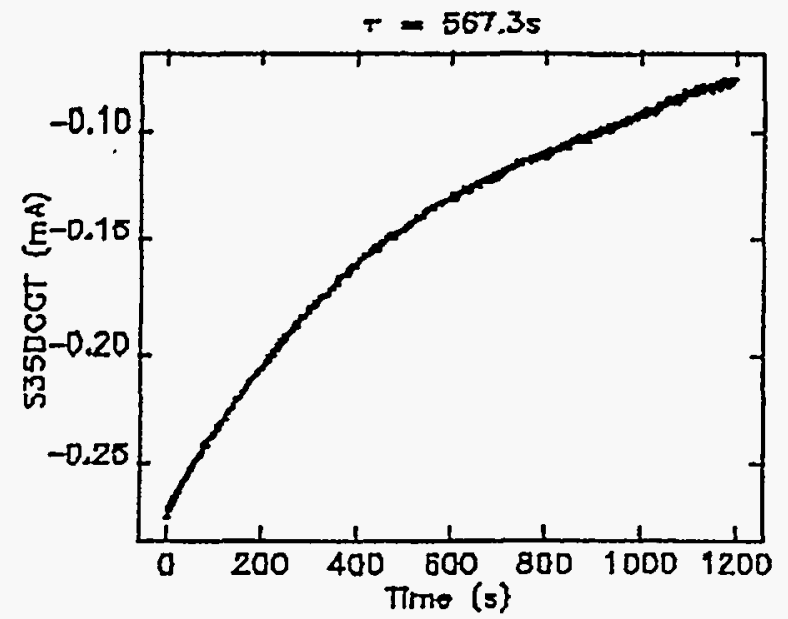

Figure 5: Storage ring PCT data

The storage ring machine protection systems (MPS) [6] are being developed to protect the accelerator components from thermal damage by missteered high-power $\mathrm{x}$-ray beams.
The system consists of a large number of fault sensors. To avoid frequent false or unnecessary machine shut-downs, many trip requests generated by the MPS subsystems require a beam intensity-based decision made in the central MPS summation box. Two independent analog signals proportional to the average beam current and another TIL digital signal indicating minimum beam current status are required to link to the central MPS logic. One analog signal is obtained by applying the PCT output to an absolute value generator that produces output voltage equal in magnitude to the PCT output voltage regardless of the beam polarities. In order to provide the second independent measurement, a new kind of storage ring average current monitor based on the ICT and gated integrator has been developed. The basic concept is to integrate all the bunches in the ring by a fast, high-precision gated integrator to obtain the accumulated total charge. This is accomplished by opening the gate window for exactly one turn (revolution time $=3.68 \mu \mathrm{s}$ ). After the gate window is closed, the final output proportional to the average current is sampled and held by a sample-and-hold amplifier which produces a DC output voltage scaled to the same range and sensitivity as the PCT. The output is updated every few turns, which offers the advantage of fast response time over the PCT. The system will be fully tested when the storage ring starts commissioning of multibunch accumulation. A full description and test results plus other design features of the MPS current monitor interface system will be presented later.

\section{CONCLUSION}

Tremendous progress has been made in commissioning the APS in the past year. The current monitor played a important role in diagnostics support to the commissioning. While the present current monitor designs have been proven sound and adequate, more improvements will be made in the areas of noise reduction and resolution enhancement.

\section{REFERENCES}

[1] X. Wang, F. Lenkszus, and E. Rotela, "Design and Commissioning of the APS Beam Charge and Current Monitors," Proc. of the 6th Accelerator Beam Instrumentation Workshop (BIW), Vancouver, B. C., Canada, 1994.

[2] K. Unser, "Design and Preliminary Tests of a Beam Intensity Monitor for LEP," Proc. of the 1989 IEEE Particle Accelerator Conference, Vol. 1, p. 71, 1989.

[3] X. Wang, "Ultrafast, High Precision Gated Integrator," Proc. of the 6th BIW, 1994.

[4] X. Wang, "Design and Initial Tests of Beam Current Monitoring Systems for the APS Transport Lines," AIP Conference Proceedings, No. 281, p. 234-241, 1992.

[5] X. Wang, M. Knott, and A. Lumpkin, "High Beam Current Shut-Off Systems in the APS linac and Low Energy Transport Line," Proc. of the 6th BIW, 1994.

[6] A. Lumpkin, et al., "Overall Design Concepts for the APS Storage Ring Machine Protection System," these proceedings. 


\section{DISCLAIMER}

This report was prepared as an account of work sponsored by an agency of the United States Government. Neither the United States Government nor any agency thereof, nor any of their employees, makes any warranty, express or implied, or assumes any legal liability or responsibility for the accuracy, completeness, or usefulness of any information, apparatus, product, or process disclosed, or represents that its use would not infringe privately owned rights. Reference herein to any specific commercial product, process, or service by trade name, trademark, manufacturer, or otherwise does not necessarily constitute or imply its endorsement, recommendation, or favoring by the United States Government or any agency thereof. The views and opinions of authors expressed herein do not necessarily state or reflect those of the United States Government or any agency thereof. 